\title{
The prediction model of moisture content's stabilization during tobacco strip drying process
}

\author{
Mingjian Zhang ${ }^{\mathrm{a}}$, Feng Huang , Qing Chen ${ }^{\mathrm{b}}$, Le Wanga, *, Haisheng Wang ${ }^{\mathrm{a}}$, Bin Li $^{\mathrm{a}}$, \\ Bin Wanga \\ ${ }^{a}$ Key Laboratory of Tobacco Processing Technology, Zhengzhou Tobacco Research Institute of \\ CNTC, Zhengzhou, China. \\ b Hua Huan International Tobacco Co., Ltd., Chuzhou, China.
}

*E-mail of the corresponding author: wangl@ztri.com.cn

\begin{abstract}
A mathematical model was established to predict the mean value and variance of tobacco strip during drying processing, based that the physical and chemical properties of tobacco strip as agriculture products show probability distributions. The results show that the model can predict the mean value of moisture content at different times, and there is a certain deviation in predicting the variance of moisture content at initial stage of drying process. However, the prediction value of the variance of tobacco strip is much more accurate while the moisture content is between $8 \%$ and $10 \%$, which is the interval of quality requirements.
\end{abstract}

Keywords: tobacco strip; drying; mean value;variance model. 


\section{Introduction}

The studies on the drying process of tobacco raw materials[1] focused on the dynamic characteristics of water migration, due to its important effect on the physical and sensory quality of tobacco products[2-3]. Moreover, the water stability was an important product quality control target in the drying process. The current researches on the dynamic drying process of tobacco materials mainly involved the prediction of the water migration law under different drying methods, temperatures, humidities and pressures through thin layer models, semi experiences and empirical models[4-6]. However, as a agricultural product, tobacco raw materials did not have the uniform physical and chemical properties, always showing widespread characteristics. Tobacco strip was constantly switched between the humidifying section and the drying section, leading to a non-equilibrium state during the dynamic processing of tobacco strip. Therefore, It is difficult to describe the production stability by the mean moisture content of tobacco strip. In this study, the Newton model was selected as the basic model for describing the drying processing of tobacco strip. Combining the approximate calculation method of the mean value and variance of multidimensional continuous random variable nonlinear function, a prediction model of water stability was finally established to describe the mean value and variance of tobacco strip's moisture content during the drying processing of tobacco strip. Moreover, the model validation was carried out by comparing with the measured experimental data.

\section{Materials and Methods}

\subsection{Materials}

The experimental tobacco samples included tobacco strips $\left(\mathrm{C}_{2} \mathrm{~F}\right.$ and $\left.\mathrm{C}_{2} \mathrm{~L}, 2015\right)$ from Fujian, Jiangxi, Hunan, Sichuan, Anhui, Henan, Jilin, Liaoning and other provinces.

The length and width of tobacco strip samples were about $2 \mathrm{~cm} \times 2 \mathrm{~cm}$, and the tobacco strips were put into a constant temperature and humidity box with $40^{\circ} \mathrm{C}$ and $80 \%$ relative humidity $(\mathrm{RH})$, equilibrated 24 hours, then were sealed in self-sealing bag.

\subsection{Measurement of tobacco strip's equilibrium moisture content}

The equilibrium moisture contents of tobacco strip samples were measured by an test device for tobacco isothermal adsorption/desorption property. The mass of samples were $1.0 \mathrm{~g}$, The gas flow rate was $4800 \mathrm{ml} / \mathrm{min}$, the drying temperature was set at $70^{\circ} \mathrm{C}$, the relative humidity was set to $30 \%$, and the drying time was $2 \mathrm{~h}$. The equilibrium moisture contents were calculated by the final mass and the initial mass of tobacco strip samples. 


\section{Prediction model of moisture content stability during tobacco strip drying process}

Although tobacco strip drying process were carried out under the same temperature and humidity treatment conditions, the initial moisture content, the balance moisture and the drying rate of the tobacco strip samples showed random distribution. Therefore, the drying model shoud be further established base on its probability distribution characteristics.

\subsection{The mathematical expectation and approximate variance}

When each factor is a continuous random variable, and the influence to the result is a nonlinear function, the expectation and variance of the random variable function can be calculated by an approximate method. The calculation method is to expand the nonlinear function of the random variable at its expected value Taylor series, abandon the high order term and calculate the expectation and variance of the random variable function. For multivariate random variables, the expression of mathematical expectation and variance is as follows:

If $X_{i} \quad(i=1,2, \cdots, \mathrm{n})$ were the independent random variables, the mathematical expectation $E\left(x_{i}\right)=\mu_{i}$, the variance $D\left(x_{i}\right)=\sigma_{i}$, and the random variable function $Z\left(x_{i}\right)=g\left(x_{1}, x_{2}, \ldots, x_{\mathrm{n}}\right)$ has two order continuous partial derivative at point $\left(\mu_{1}, \mu_{2}, \ldots, \mu_{\mathrm{n}}\right)$, then the mathematical expectation

$$
E(Z) \approx g\left(\mu_{1}, \mu_{2}, \ldots, \mu_{n}\right)+\frac{1}{2} \sum_{i=1}^{n} \frac{\partial^{2} g}{\partial x_{i}^{2}} \sigma_{i}^{2}
$$

And the approximate variance

$$
D(Z) \approx \frac{1}{2} \sum_{i=1}^{n}\left(\frac{\partial g}{\partial x_{i}}\right)^{2} \sigma_{i}^{2}
$$

\subsection{Newton drying model based on multidimensional continuous random variables}

The Newton model was used as the basic drying model, and the decision coefficient $\left(R^{2}\right)$ and the chi square $\left(\chi^{2}\right)$ were used for the evaluation of the model. For Newton model, the drying rate is proportional to the moisture content.

$$
\begin{aligned}
& \frac{d x}{d t}=-k\left(x-x_{e}\right) \\
& x(0)=x_{0}
\end{aligned}
$$


In the upper formula, $x, k, x_{e}$ and $x_{0}$ respectively indicate the mean value of the moisture content(db.), drying rate constant, equilibrium moisture content(db.) and initial moisture content(db.) of tobacco strips. The relationship between water and time is obtained as follows by solving the above equations.

$$
x(t)=\left(x_{0}-x_{e}\right) \exp (-k t)+x_{e}
$$

During the actual drying process of tobacco strips, $\sigma_{k}^{2}, \sigma_{e}^{2}$ and $\sigma_{x}^{2}$ respectively indicate the variance value of drying rate constant, equilibrium moisture content(db.) and initial moisture content $(\mathrm{db}$.) of tobacco strips. As the drying rate constant, equilibrium moisture content(db.) and initial moisture content(db.) of tobacco strips were independent, The moisture content's mean and variance valuse of tobacco strips at $t$ time could be calculated as follows as a mean and variance of a random variable function.

$$
\begin{aligned}
& E[x(t)] \approx\left[\left(x_{0}-x_{e}\right) \exp (-k t)+x_{e}\right]+\frac{1}{2}\left(x_{0}-x_{e}\right) \exp (-k t) t^{2} \sigma_{k}^{2} \\
& D[x(t)] \approx \exp (-2 k t)\left[\sigma_{x}^{2}+\left(x_{0}-x_{e}\right)^{2} t^{2} \sigma_{k}^{2}+\sigma_{e}^{2}\right]-2 \exp (-k t) \sigma_{e}^{2}+\sigma_{e}^{2}
\end{aligned}
$$

From the formula (5) and (6), the mean and variance values of moisture content during the actual drying process could be calculated by mean values of Newton model parameters, and the error decreased with exponential function. Moreover, the variance of moisture content decreased exponentially with the increasing drying time, and finally decreased to the variance of equilibrium moisture content $\left(\sigma_{e}^{2}\right)$.

\section{Results and discussion}

\subsection{The drying kinetic curves of tobacco strips}

The drying kinetic curves of 83 types of tobacco strips samples from different localities were obtained under the drying temperature of $70^{\circ} \mathrm{C}$ and $30 \% \mathrm{RH}(\mathrm{Fig}$. 1). The above results showed that, the initial moisture contents of the sample were distributed in an interval after the same pretreatment, and the moisture contents changed at different drying rates. Moreover, the moisture of the tobacco strips rapidly decreased at the initial stage during a fast drying stage. Then the drying rates reduced gradually leading to a slow drying process, and finally reached the equilibrium moisture contents under the drying condition. The distribution of the equilibrium moisture contents was more concentrated. The moisture content decreased with exponential trend during the whole drying process. 


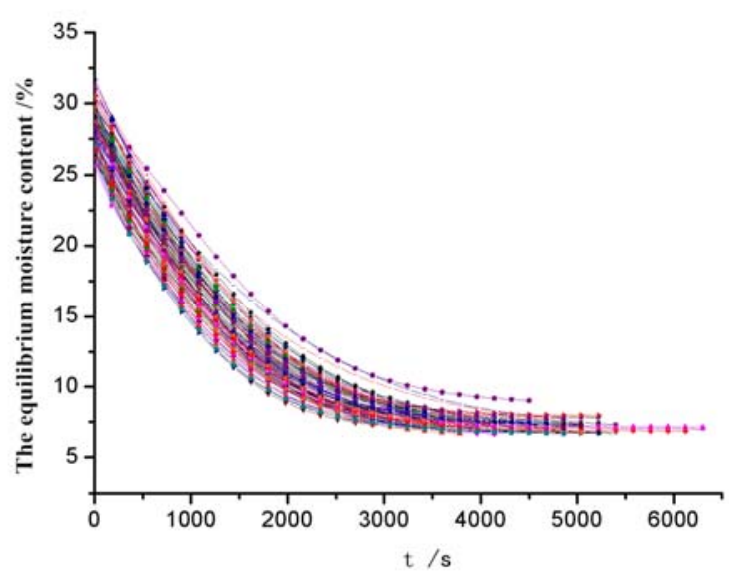

Fig. 1 The drying kinetic curves of tobacco strips.

\subsection{Probability statistics}

The frequency $(\mathrm{H})$ distribution of the initial moisture content, equilibrium moisture content and drying rate constant $(\mathrm{k})$ of Newton model under the same drying conditions were shown in Fig. 3. The results indicated that the initial moisture content, equilibrium moisture content and drying rate constant had the same normal distribution form and all the decision coefficients $\left(R^{2}\right)$ of Newton model were higher than 0.985 , showing that the Newtong model was suitable for calculating these drying process. The correlation analysis of the initial moisture content, equilibrium moisture content and drying rate constant $(\mathrm{k})$ of Newton model was shown in Table 1. The results showed that these above factors had weak positive correlation and negative correlation, according with the model requirements for independent variables.
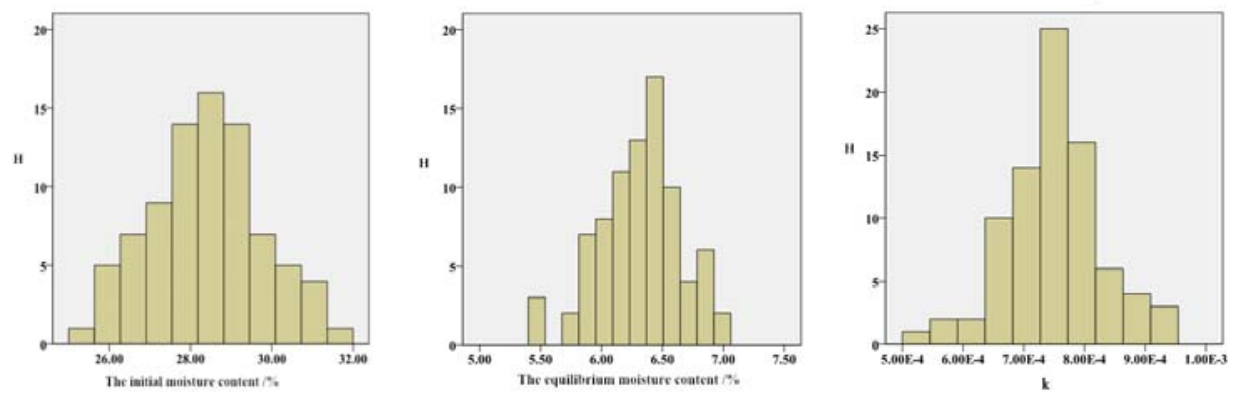

Fig. 2 The frequency $(\mathrm{H})$ distribution of the initial moisture content, equilibrium moisture content and drying rate constant $(k)$ of Newton model under the same drying conditions 
Table 1. The correlation analysis

\begin{tabular}{llll}
\hline Factor & $\mathbf{k}$ & $\begin{array}{l}\text { The } \\
\text { moisture content }\end{array}$ & $\begin{array}{l}\text { initial equilibrium } \\
\text { moisture content }\end{array}$ \\
\hline $\mathrm{k}$ & 1 & & \\
The initial moisture content & -0.296 & 1 & 1 \\
The equilibrium moisture content & -0.457 & 0.545 & \\
\hline
\end{tabular}

\subsection{Model validation}

The predicted moisture content's mean and variance values during the drying processing of tobacco strips were compared with the measured values in Fig. 3. The results showed that the difference between the predicted and measured mean values of tobacco strips moisture content (db.) was relatively small, which indicated that this model could predict the mean value of the whole drying process. Moreover, the difference between the predicted and measured variance values of tobacco strips moisture content $(\mathrm{db}$.) increased first and then decreased, while the predicted variances obtained by approximate method were a little smaller than the actual statistical value, due to the nonlinearity of Newton model and a certain correlation of these model parametes. It was worth noting that the target moisture (db.) in the typical tobacco strips drying processing were between $8.7 \%$ and $11.1 \%$, which was included in the critical area of free water and combined water. In this moisture content interval, the difference between predicted values and measured values became rather small, which indicated that the predicted model was reliable between the moisture content interval of the drying process quality requirement.
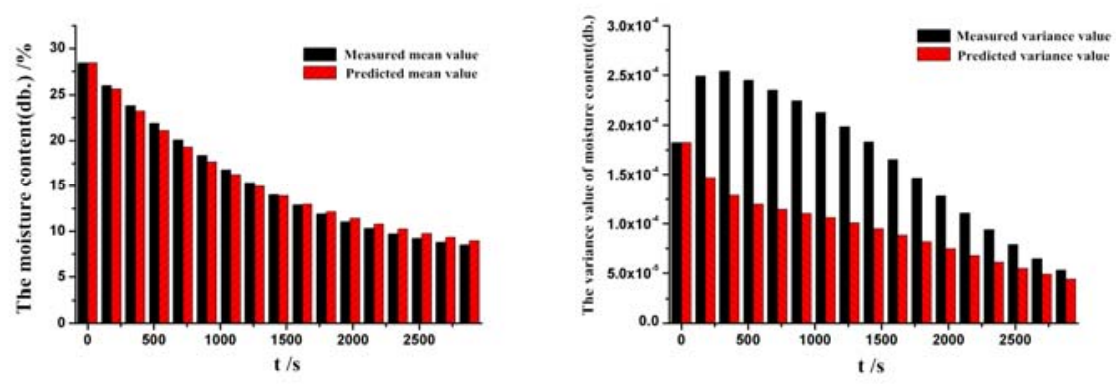

Fig. 3 The measured and predicted moisture content's mean and variance values during the drying processing of tobacco strips. 


\section{Conclusion}

Combining the approximate calculation method of the mean value and variance of multidimensional continuous random variable nonlinear function, a mean and variance prediction model of the tobacco strips' moisture contents during the drying process was established base on Newton model. Moreover, the distribution and linear correlation between the initial moisture content, equilibrium moisture content and drying rate constant were discussed, which was consistent with the application requirements of the model. The predicted model showed good performance on the prediction of mean moisture contents during the whole drying process. The prediction of moisture contents' variance had a certain deviation in the initial drying stage, while the predicted variances were more accurate between the moisture content $(\mathrm{db}$.) interval $(8.7 \% \sim 11.1 \%)$ of the drying process quality requirement, which was included in the critical area of free water and combined water.

\section{References}

[1] Legros, R.; Millington, CA; Clift, R. Drying of tobacco particles in a mobilized bed. Drying Technology 1994, 12 (3), 517-543.

[2] Wang H.; Xin H.; Liao Z.; et al. Study on the effect of cut tobacco drying on the pyrolysis and combustion properties. Drying technology 2014, 32(2): 130-134.

[3] Zhu W. K.; Wang Y.; Chen L. Y.; et al. Effect of two-stage dehydration on retention of characteristic flavor components of flue-cured tobacco in rotary dryer. Drying technology 2016, 34(13): 1621-1629.

[4] Geng F.; Xu D.; Yuan Z.; et al. Numerical simulation on fluidization characteristics of tobacco particles in fluidized bed dryers. Chemical Engineering Journal 2009, 150(23): 581-592.

[5] Feng H.; Qing C.; Le Y. U.; et al. Study on the thin layer kinetics models of humidifying and drying of tobacco strips. Acta Tabacaria Sinica 2014, 20(6): 34-40.

[6] Xin Y. N.; Zhang J. W.; Li B. Drying kinetics of tobacco strips at different air temperatures and relative humidities. Journal of Thermal Analysis and Calorimetry 2018, 132(2): 1347-1358. 